\title{
Immunotherapy and molecular role of T-cell in PD-1 antibody treated resectable lung cancer patients
}

\author{
Samaresh Sau ${ }^{1}$, Arun K. Iyer ${ }^{1,2}$ \\ ${ }^{1}$ Use-inspired Biomaterials \& Integrated Nano Delivery (U-BiND) Systems Laboratory, Department of Pharmaceutical Sciences, Eugene Applebaum \\ College of Pharmacy and Health Sciences, Wayne State University, Detroit, MI, USA; ${ }^{2}$ Molecular Imaging Program, Barbara Ann Karmanos Cancer \\ Institute, Wayne State University, School of Medicine, Detroit, MI, USA \\ Correspondence to: Samaresh Sau, PhD; Arun K. Iyer, PhD. Use-inspired Biomaterials \& Integrated Nano Delivery (U-BiND) Systems Laboratory, \\ Department of Pharmaceutical Sciences, Eugene Applebaum College of Pharmacy and Health Sciences, Wayne State University, 259 Mack Ave, \\ Room 3601, Detroit, MI 48201, USA. Email: samaresh.sau@wayne.edu; arun.iyer@wayne.edu. \\ Provenance: This is an invited Editorial commissioned by the Section Editor Xiaozheng Kang (Department of Thoracic Surgery, Beijing Cancer \\ Hospital, Peking University, Beijing, China). \\ Comment on: Forde PM, Chaft JE, Smith KN, et al. Neoadjuvant PD-1 Blockade in Resectable Lung Cancer. N Engl J Med 2018;378:1976-86.
}

Submitted Jun 26, 2018. Accepted for publication Jul 09, 2018.

doi: $10.21037 /$ jtd.2018.07.66

View this article at: http://dx.doi.org/10.21037/jtd.2018.07.66

In the tumor microenvironment, overexpression of check point molecules, such as program death ligand-1 (PD-L1) functions as a protector against the immune surveillance by T-cell through the interaction with program death-1 (PD-1) receptor (1). It has been found that $\mathrm{PD}-1$ overexpressed on a variety of tumor-killing immune cells, such as monocytes, macrophages, cytotoxic CD8+ T cells, and dendritic cells has an active role in hijacking the antitumor immune response (2). Therefore, inhibition of PD-1 and PD-L1 interaction would resurrect the tumor-killing effect of CD8+ T cells $(3,4)$. The immunosuppressive regulatory $\mathrm{T}$ cells (Treg, CD4+ Foxp3+) also overexpress PD-1 receptor that favors immune suppression of tumor and negatively regulates $\mathrm{CD} 8+\mathrm{T}$ cells $(5,6)$. Thus, the use of $\mathrm{PD}-1$ inhibitors not only reactivate the function of CD8+ T cell but also downmodulate the function of Treg and tumorassociated macrophage (TAM) cells through inhibition of mammalian target of rapamycin (mTOR)-Akt and Stat3 signaling cascade (7) (Figure 1). Several clinical trials are ongoing using PD-1/PD-L1 therapies for NSCLC (e.g., nivolumab, pembrolizumab, atezolizumab, and durvalumab) (Figure 2). The use of immunotherapy has been a game changer in comparison to conventional cancer therapy as they can be personalized for individual therapy or can be combined with chemotherapeutics, targeted therapeutics and nanomedicines (9-13).

In the $N$ Eng $7 \mathrm{Med}$ article, Forde et al. described a novel pilot study of 22 patients treated with neoadjuvant PD-1 inhibitor treatment to lung cancer (LC), followed by surgical resection of the leftover tumors. The PD-1 inhibitor, Nivolumab that is clinically approved for renal cell carcinoma, metastatic melanoma, hepatocellular carcinoma has been first-time tested in resectable LC. In this study adult patients with stage I, II, IIIA LC were treated with nivolumab by intravenous administration at a dose of $3 \mathrm{mg}$ per $\mathrm{kg}$ of body weight every 2 weeks, with surgery planned approximately 4 weeks after the first dose (14). The safety, pathological response, molecular role of T-cell activation, the mutational burden in the tumor was evaluated in resected tumor samples. This regimen resulted in a few manageable side effects and induced an overall $45 \%$ pathological response to patients as evaluated by immunohistological analysis.

\section{Patient selection, therapeutic outcome and molecular analysis of the tumor}

The study has been initiated from August 2015 through October 2016 with 22 patents and all patients received at least one single dose of nivolumab. Among the 22 patients, 21 patients were eligible for the further study and underwent surgery for tumor resection. The patients had different phenotypes of LC including 62\% (13 patients) with lung adenocarcinoma and 29\% (29 patients) with 

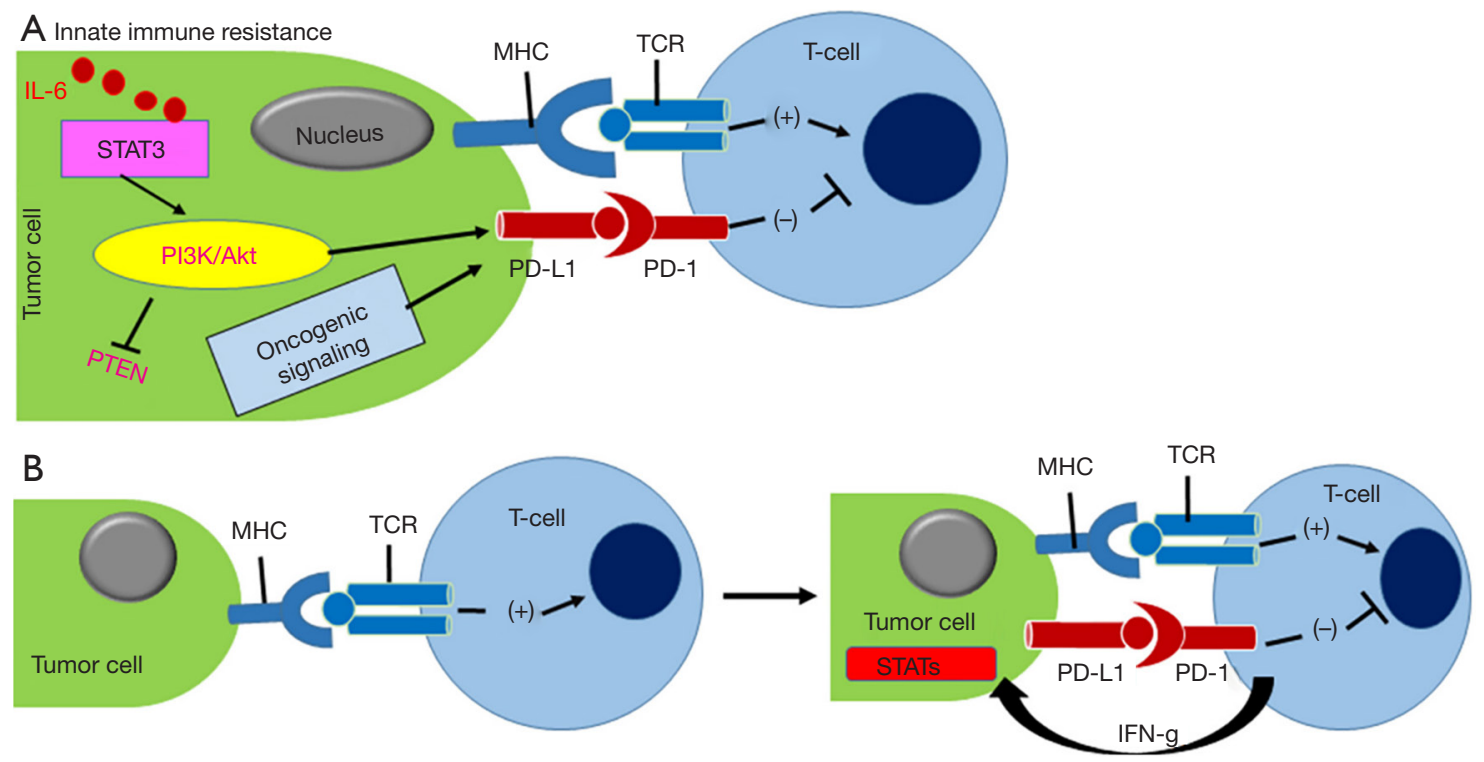

Figure 1 (A) Innate immune resistance is driven by activation of PI3K/Akt kinase and IL-6/STAT3 oncogenic signaling that up-modulate PDL-1 protein expression in tumor cells, resulting PD-1/PD-L1 complexation; (B) the adaptive immune resistance of cancer cells is the outcome of INF- $\gamma$ responded PD-L1 expression. MHC and TCR interaction helps T-cell activation. Figure and legend were adopted with permission from reference (1). PD-L1, program death ligand-1; PD-1, program death-1; MHC, major histocompatibility complex; TCR, T-cell receptor.

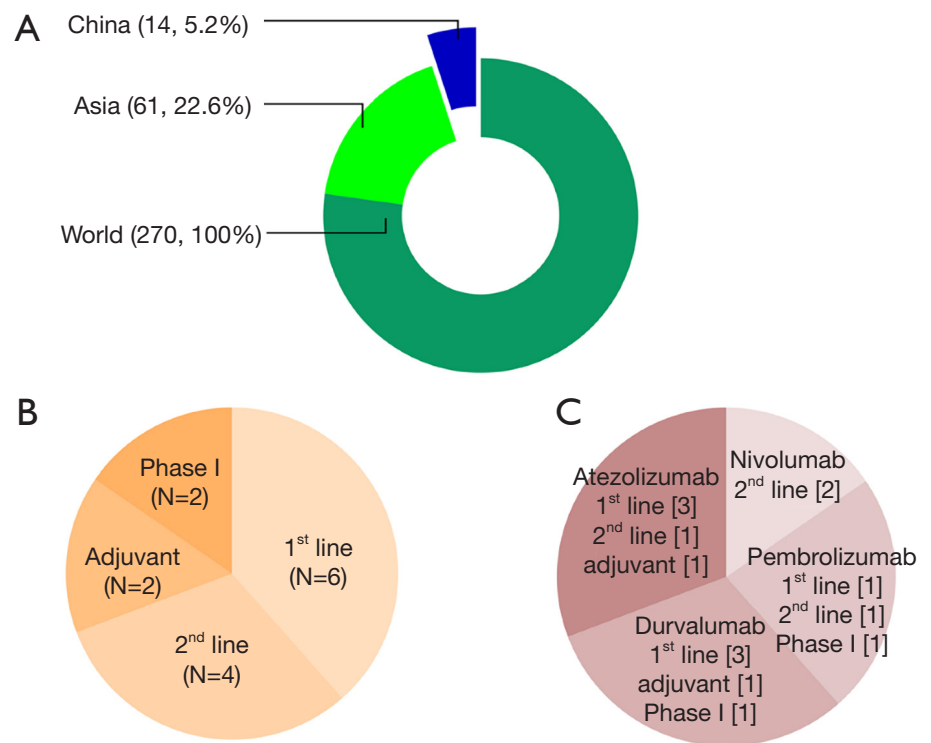

Figure 2 Ongoing international clinical trials including Chinese patients. (A) Between January 01, 2013, and April 06, 2017, there were 270 clinical trials of anti-PD-1/PD-L1 inhibitors for NSCLC that were registered on ClinicalTrials.gov. Among the 270 studies, 61 studies were performed in East Asia and 14 studies were performed in China (12 multinational trials and 2 trials that only evaluated Chinese patients); (B) the 14 clinical trials included six first-line studies, four second-line studies, two adjuvant therapy studies, and two phase I studies for only Chinese patients; $(C)$ the classification of clinical trials in China according to the therapeutic agent, which includes nivolumab, pembrolizumab, atezolizumab, and durvalumab. The image and legend were adopted with permission from reference (8). PD-L1, program death ligand-1; PD-1, program death-1. 
squamous cell carcinoma. Ten percent ( 2 patients) had other types of pleomorphic carcinoma as determined by histological diagnosis. This study is supportive of the observation made by Yang et al., where authors found the mutation of BRAF, ROS1, KRAS and EGFR genes and the rearrangement of the $E M L 4-A L K$ gene in 119 lung cancer patients in China (15). In neoadjuvant nivolumab study, it has been reported that 18 patients $(86 \%)$ were either former or current smokers and 3 patients (14\%) never smoked. All the enrolled patients including 11 females (52\%) and 10 males $(48 \%)$ were above 60 years and had variability in tumor stages including 4 patients with stage I (19\%), 10 patients (48\%) with stage II and 2 patients (10\%) stage IIIA based on clinical disease condition.

The response of nivolumab on the patients was determined by computed tomography (CT) analysis and hemotoxin and eosin (H\&E) staining after two preoperative doses of nivolumab. The data suggest that out of the 21 patients $18(86 \%)$ had stable disease, 2 patients (10\%) had a partial response, and $1(5 \%)$ had disease progression. Among the 21 patients, 20 patients had undergone surgical resection of tumor and postoperative diagnosis after 12 months of surgery suggested that 16 patients (80\%) were alive and disease free. The pathological response by $\mathrm{H} \& \mathrm{E}$ staining was done in 9 out of 20 patients. The histological analysis of the tumors isolated from 3 patients out of 9 revealed $100 \%$ pathological response that was accompanied with no invasion of cell boundaries, no anaplasia, and no sign of mitotic nucleus as compared to nivolumab untreated tumor biopsy $(16,17)$. To further evaluate the effect of nivolumab in patients multiplex immunofluorescence analyses were performed in tumor biopsy samples to ascertain the role of PD-L1, PD-1, tumor-associated CD68+ macrophages, FoxP3+ regulatory T cells, and CD8+ $\mathrm{T}$ cells. The biopsy specimen data revealed that there were few PD-L1 positive intratumoral macrophages and the expression of PD-L1 and PD-1 were in close proximity to each other. The tumors were rich with infiltrated CD8+ and PD-1+ immune cells and some of the infiltrating immune cells expressed PD-L1 that suggests their role in inducing adaptive immune resistance to the tumor. Overall, the pathological response data suggests that nivolumab treatment was beneficial in both $\mathrm{PD}-\mathrm{L} 1+$ positive and PD-L1- negative tumors.

In this study, the whole exome sequencing was also performed with the resected tumor of 12 patients and the data indicate several valuable information such as, a median of 92 somatic mutations (range is 5 to 366) per tumor and
TP53, KRAS, CDKN2A, ARID1A, and NOTCH1 were the mutated genes that drive that development of LC. Authors have reported that there is no alteration of immune-related gene of interest, including PDCD1, CTLA4, and $\mathcal{F A K 1 / 2 .}$ The dynamic antitumor response of T-cells in PD-1 therapy depends on the abundance of tumor-specific T-cell clones and the function of complementarity-determining regions (CDRs) of $\mathrm{T}$ cells in the tumor milieu. CDR is the $\mathrm{F}^{\mathrm{ab}}$ domain of $\mathrm{T}$ cell receptors, where the antigens bind $(18,19)$. The deep sequencing of T-cell receptor- $\beta$ chain CDR3 region was performed to examine the effect of nivolumab treatment in accumulating different clones of T-cells in tumor and peripheral blood of 3 patients with the major pathological response and 6 patients without a major response $(20,21)$. The data showed that the tumor with a greater pathological response had a higher number of $\mathrm{T}$ cells both in intratumor and peripheral regions. Authors have identified mutation associated neoantigens of $\mathrm{T}$ cells to explain the antitumor immunity of PD-1 blockage. The antigen-recognition assay was performed with T-cell clones after peptide stimulation with the use of 47 algorithm-prediction. The frequency of $\mathrm{T}$ cell in nivolumab treated tumor is significantly higher in case of "tumor before the resection" as compared to "resection normal lung" or "resection involved lymph node" or "resection uninvolved lymph node". The significant findings of this study where the pathological response of nivolumab treated patients on early-stage lung cancer that was associated with increased tumor mutational burden as well as the significant response to PD-1 blockade in patients with advanced resectable NSCLC. One of the limitations of this study was the small patient numbers that could skew the validity of the improvement of the neoadjuvant treatment. Thus, larger studies are required with neoadjuvant as a single therapy and in combination with other chemotherapeutic drugs. The through safety analysis and immune-related adverse events (IRAE) evaluation is required for curing the high gene mutation containing lung cancer.

\section{Acknowledgements}

Wayne State Startup Funds.

\section{Footnote}

Conflicts of Interest: The authors have no conflicts of interest to declare. 


\section{References}

1. Alsaab HO, Sau S, Alzhrani R, et al. PD-1 and PDL1 Checkpoint Signaling Inhibition for Cancer Immunotherapy: Mechanism, Combinations, and Clinical Outcome. Front Pharmacol 2017;8:561.

2. Sun Z, Fourcade J, Pagliano O, et al. IL10 and PD-1 Cooperate to Limit the Activity of Tumor-Specific CD8+ T Cells. Cancer Res 2015;75:1635-44.

3. Zou W, Chen L. Inhibitory B7-family molecules in the tumour microenvironment. Nat Rev Immunol 2008;8:467-77.

4. Butte MJ, Keir ME, Phamduy TB, et al. Programmed death-1 ligand 1 interacts specifically with the B7-1 costimulatory molecule to inhibit $\mathrm{T}$ cell responses. Immunity 2007;27:111-22.

5. Sau S, Alsaab HO, Bhise K, et al. Multifunctional nanoparticles for cancer immunotherapy: A groundbreaking approach for reprogramming malfunctioned tumor environment. J Control Release 2018;274:24-34.

6. Francisco LM, Sage PT, Sharpe AH. The PD-1 pathway in tolerance and autoimmunity. Immunol Rev 2010;236:219-42.

7. Ohaegbulam KC, Assal A, Lazar-Molnar E, et al. Human cancer immunotherapy with antibodies to the PD-1 and PD-L1 pathway. Trends Mol Med 2015;21:24-33.

8. Liu SY, Wu YL. Ongoing clinical trials of PD-1 and PDL1 inhibitors for lung cancer in China. J Hematol Oncol 2017;10:136.

9. Pardoll DM. The blockade of immune checkpoints in cancer immunotherapy. Nat Rev Cancer 2012;12:252-64.

10. Wang Z, Sau S, Alsaab HO, et al. CD44 directed nanomicellar payload delivery platform for selective anticancer effect and tumor specific imaging of triple negative breast cancer. Nanomedicine 2018;14:1441-54.

11. Sau S, Agarwalla P, Mukherjee S, et al. Cancer cellselective promoter recognition accompanies antitumor effect by glucocorticoid receptor-targeted gold

Cite this article as: Sau S, Iyer AK. Immunotherapy and molecular role of T-cell in PD-1 antibody treated resectable lung cancer patients. J Thorac Dis 2018;10(8):4682-4685. doi: 10.21037/jtd.2018.07.66 nanoparticle. Nanoscale 2014;6:6745-54.

12. Sau S, Mondal SK, Kashaw SK, et al. Combination of cationic dexamethasone derivative and STAT3 inhibitor (WP1066) for aggressive melanoma: a strategy for repurposing a phase I clinical trial drug. Mol Cell Biochem 2017;436:119-36.

13. Sau S, Tatiparti K, Alsaab HO, et al. A tumor multicomponent targeting chemoimmune drug delivery system for reprograming the tumor microenvironment and personalized cancer therapy. Drug Discov Today 2018;23:1344-56.

14. Forde PM, Chaft JE, Smith KN, et al. Neoadjuvant PD-1 Blockade in Resectable Lung Cancer. N Engl J Med 2018;378:1976-86.

15. Yang $Y$, Yin $W$, He $W$, et al. Phenotype-genotype correlation in multiple primary lung cancer patients in China. Sci Rep 2016;6:36177.

16. Shoshan-Barmatz V, Bishitz Y, Paul A, et al. A molecular signature of lung cancer: potential biomarkers for adenocarcinoma and squamous cell carcinoma. Oncotarget 2017;8:105492-509.

17. Cheriyan VT, Alsaab HO, Sekhar S, et al. A CARP1 functional mimetic loaded vitamin E-TPGS micellar nano-formulation for inhibition of renal cell carcinoma. Oncotarget 2017;8:104928-45.

18. Sau S, Alsaab HO, Kashaw SK, et al. Advances in antibody-drug conjugates: A new era of targeted cancer therapy. Drug Discov Today 2017;22:1547-56.

19. Leem J, de Oliveira SHP, Krawczyk K, et al. STCRDab: the structural T-cell receptor database. Nucleic Acids Res 2018;46:D406-12.

20. Shindo T, Kitaura K, Ureshino H, et al. Deep sequencing of the $\mathrm{T}$ cell receptor visualizes reconstitution of $\mathrm{T}$ cell immunity in mogamulizumab-treated adult $\mathrm{T}$ cell leukemia. Oncoimmunology 2017;7:e1405204.

21. Srivastava SK, Robins HS. Palindromic nucleotide analysis in human T cell receptor rearrangements. PLoS One 2012;7:e52250. 